\title{
Diagnostic Impact of Intracranial Vessel Wall MRI in 205 Patients with Ischemic Stroke or TIA
}

\author{
(D).D. Schaafsma, (D) S. Rawal, (D).M. Coutinho, (D). Rasheedi, (DD.J. Mikulis, (D) C. Jaigobin, (D)F.L. Silver, and (DD.M. Mandell
}

\begin{abstract}
BACKGROUND AND PURPOSE: Secondary prevention of ischemic stroke depends on determining the cause of the initial ischemic event, but standard investigations often fail to identify a cause or identify multiple potential causes. The purpose of this study was to characterize the impact of intracranial vessel wall MR imaging on the etiologic classification of ischemic stroke.
\end{abstract}

MATERIALS AND METHODS: This was a single-center, retrospective study of 205 consecutive patients who were referred for vessel wall MR imaging to clarify the etiology of an ischemic stroke or TIA. An expert panel classified stroke etiology before and after incorporating vessel wall MR imaging results using a modified Trial of Org 10172 in Acute Stroke Treatment system. We measured the proportion of patients with an altered etiologic classification after vessel wall MR imaging.

RESULTS: The median age was 56 years (interquartile range $=44-67$ years), and 51\% (106/205) of patients were men. Vessel wall MR imaging altered the etiologic classification in 55\% (112/205) of patients. The proportion of patients classified as having intracranial arteriopathy not otherwise specified decreased from $31 \%$ to $4 \%(64 / 205$ versus $9 / 205 ; P<.001)$ and the proportion classified as having intracranial atherosclerotic disease increased from $23 \%$ to $57 \%(48 / 205$ versus $116 / 205 ; P<.001)$. Conventional work-up classification as intracranial arteriopathy not otherwise specified was an independent predictor of vessel wall MR imaging impact $(\mathrm{OR}=8.9$; 95\% CI , 3.0-27.2). The time between symptom onset and vessel wall MR imaging was not a predictor of impact.

CONCLUSIONS: When vessel wall MR imaging is performed to clarify the etiology of a stroke or TIA, it frequently alters the etiologic classification. This is important because the etiologic classification is the basis for therapeutic decision-making.

ABBREVIATION: $V W$ = vessel wall

S econdary prevention of ischemic stroke depends on determining the cause of the initial stroke or TIA. However, for $25 \%$ of patients, standard investigations fail to identify a cause; $^{1}$ and investigations sometimes identify multiple potential causes.

Conventional imaging of the intracranial arteries (using CTA, MRA, or conventional angiography) shows the contour of the arterial lumen, but not the arterial wall itself. This approach fails to detect nonstenotic intracranial atherosclerotic disease $e^{2-4}$ and to

Received May 9, 2019; accepted after revision July 24.

From the Division of Neurology, Department of Medicine (J.D.S., J.R., C.J., F.L.S.), and Division of Neuroradiology, Department of Medical Imaging (S.R., J.M.C., D.J.M., D.M.M.), University Health Network, Toronto, Ontario, Canada; and Department of Neurology (I.M.C.), Academic Medical Center, Amsterdam, the Netherlands.

This study was supported by the Ontario Academic Health Science Centres Innovation Fund.

Please address correspondence to Daniel Mandell, MD, PhD, Medical Imaging, $3 \mathrm{MCL}-426$, Toronto Western Hospital, 399 Bathurst St, Toronto, ON M5T2S8,

Canada; e-mail: danny.mandell@uhn.ca

Indicates article with supplemental on-line table.

http://dx.doi.org/10.3174/ajnr.A6202 differentiate disorders such as vasculitis and reversible cerebral vasoconstriction syndrome. ${ }^{5}$ It also incompletely characterizes disease activity, contributing to uncertainty about whether a particular vascular abnormality is incidental or the culprit etiology.

High-resolution vessel wall (VW) MR imaging is an adjunct to conventional vascular imaging. VW-MR imaging shows the arterial wall directly and enables the diagnosis of nonstenotic arterial disease, ${ }^{2-4}$ differentiation of diseases that have a similar appearance on conventional vascular imaging, ${ }^{6-8}$ and assessment of vascular disease activity. ${ }^{9}$ Studies have described the VW-MR imaging appearance of several stroke etiologies ${ }^{6,9,10}$ and measured the diagnostic accuracy of specific vessel wall findings. ${ }^{8,11,12}$ There are practice guidelines for clinical use of VWMR imaging, 9,13 and the technique has been increasingly adopted on a clinical basis.

However, there remains a broader question. When intracranial VW-MR imaging is performed to clarify the etiology of a stroke or TIA, how often and in what circumstances does this supplementary examination have an impact on the etiologic classification? 
Table 1: Framework for diagnostic categorization of vessel wall MR imaging findings ${ }^{\mathrm{a}}$

\begin{tabular}{|c|c|}
\hline VW-MR Imaging Category & Findings \\
\hline Normal & $\begin{array}{l}\text { Arterial wall is thin or imperceptible; no wall enhancement other } \\
\text { than } \pm \text { proximal intracranial internal carotid and vertebral } \\
\text { arteries attributed to vasa vasorum }\end{array}$ \\
\hline $\begin{array}{l}\text { Intracranial atherosclerotic } \\
\text { disease }\end{array}$ & $\begin{array}{l}\text { Arterial wall is focally, eccentrically thickened; T2 prolongation } \pm \\
\text { enhancement immediately adjacent to the lumen and T2 } \\
\text { shortening within the wall more peripherally; markers of disease } \\
\text { activity include plaque enhancement and intraplaque } \\
\text { hemorrhage }\end{array}$ \\
\hline Arterial dissection & $\begin{array}{l}\text { Arterial wall is eccentrically thickened; thickened wall has the } \\
\text { signal characteristics of blood products; marker of disease } \\
\text { activity is intramural signal characteristics of acute or subacute } \\
\text { blood product }\end{array}$ \\
\hline Vasculitis & $\begin{array}{l}\text { Arterial wall is concentrically, homogeneously thickened and } \\
\text { enhancing }\end{array}$ \\
\hline $\begin{array}{l}\text { Reversible cerebral } \\
\text { vasoconstriction } \\
\text { syndrome }\end{array}$ & $\begin{array}{l}\text { Arterial wall is concentrically, homogeneously thickened with no } \\
\text { (or mild) enhancement }\end{array}$ \\
\hline
\end{tabular}

${ }^{a}$ Adapted from the consensus recommendations of the Vessel Wall Imaging Study Group of the American Society of Neuroradiology. ${ }^{9}$

To answer this question, we studied 205 consecutive patients who had VW-MR imaging performed to clarify the etiology of a stroke or TIA. We interpreted the VW-MR imaging according to consensus guidelines and used expert-panel adjudication to characterize the impact of VW-MR imaging on the etiologic classification.

\section{MATERIALS AND METHODS Patients}

This was a single-center, retrospective study at the University Health Network, Toronto, Ontario, Canada. We included consecutive patients referred from the hospital stroke service between 2006 and 2014 for intracranial VW-MR imaging to clarify the etiology of an ischemic stroke or TIA. We excluded patients scanned after 2014 to enable a separate analysis of long-term clinical follow-up. The institutional review board approved the study.

\section{High-Resolution Intracranial Vessel Wall MR Imaging}

VW-MR imaging on a 3T MR imaging system (Signa HDx; GE Healthcare, Milwaukee, Wisconsin) with an 8-channel receiveonly head coil included time-of-flight MRA of the intracranial arteries (3D with FOV $=22 \times 22 \mathrm{~cm}$, acquired matrix $=512 \times$ 512 , acquired section thickness $=1 \mathrm{~mm}$, section overlap $=50 \%$, 145 slices, $\mathrm{TR}=21 \mathrm{~ms}$, TE $=2.7 \mathrm{~ms}$ ), a T2-weighted VW-MR imaging sequence $(2 \mathrm{D}$ fast spin-echo with $\mathrm{FOV}=22 \times 22 \mathrm{~cm}$, acquired matrix $=512 \times 512$, section thickness $=2 \mathrm{~mm}$, no interslice gap, acquired voxel $=0.4 \times 0.4 \times 2.0 \mathrm{~cm}, 15-25$ slices, $\mathrm{TR}=3250 \mathrm{~ms}, \mathrm{TE}=89 \mathrm{~ms}$ ), and a T1-weighted VW-MR imaging sequence (single inversion recovery-prepared, 2D fast spinecho with identical voxel dimensions, $\mathrm{TR}=2263 \mathrm{~ms}$, $\mathrm{TI}=$ $860 \mathrm{~ms}$, TE $=13 \mathrm{~ms}$ ) before and immediately after a 5-mL intravenous injection of gadobutrol (Gadovist; Bayer Schering Pharma, Berlin, Germany). Each VW-MR imaging sequence took 3-7 minutes, depending on the number of slices. A neuroradiologist monitored each examination to target the vessels of interest in both short- and long-axis planes, which were axial, sagittal, or coronal planes or obliques of these depending on the orientation of the vessels of interest. When conventional vascular imaging findings were normal, the VW-MR imaging target was the vessels supplying the territory of the ischemic event.

\section{Panel Adjudication Stage 1: Conventional Work-Up}

The panel included 2 neurologists with subspecialization in stroke, and 2 neuroradiologists with expertise in cerebrovascular disease. A stroke neurologist blinded to VWMR imaging results reviewed the clinical history and physical examination notes, laboratory results, and conventional imaging reports for each patient. The neurologist categorized stroke etiology for each patient using a modification of the Trial of Org 10172 in Acute Stroke Treatment (TOAST) ${ }^{14}$ categories. We used the TOAST categories of cardioembolism, small-artery occlusion, other determined etiology, and undetermined etiology. We limited use of the TOAST category "large artery atherosclerosis" to the cervical arteries and categorized intracranial atherosclerotic disease separately. We supplemented these categories with 4 additional categories: intracranial arterial dissection, vasculitis, reversible cerebral vasoconstriction syndrome, and intracranial arteriopathy not otherwise specified. The undetermined category includes the subcategories negative evaluation, incomplete evaluation, and $\geq 2$ causes identified. The second stroke neurologist independently categorized stroke etiology for 50 randomly selected patients in the study to assess interobserver variability.

\section{Panel Adjudication Stage 2: Incorporating VW-MR Imaging}

A neuroradiologist reviewed the VW-MR imaging for each patient and flagged any examinations that were completely nondiagnostic due to poor technical quality. The neuroradiologist categorized VW-MR imaging findings using the framework described in the Consensus Recommendations of the Vessel Wall Imaging Study Group of the American Society of Neuroradiology, ${ }^{9}$ which is summarized in Table 1 . We followed the recommendations for interpretation of VW-MR imaging, ${ }^{9}$ including the need to confirm vessel wall findings in multiple planes and with multiple tissue weightings, with accurate determination of the inner and outer boundaries of the vessel wall, to confirm that vessel wall findings were indeed within the vessel wall and not thrombus within the lumen or outside the vessel. Using the same framework, the neuroradiologist also recorded whether there were VW-MR imaging findings to suggest active rather than quiescent disease. A second neuroradiologist independently categorized VW-MR imaging findings for 50 randomly selected patients in the study to assess interobserver variability. 
Table 2: Conventional investigations performed to determine the etiology of TIA or stroke in 205 patients

\begin{tabular}{lc}
\hline \multicolumn{1}{c}{ Investigation } & $\begin{array}{c}\text { Proportion of } \\
\text { Patients }\end{array}$ \\
\hline Brain imaging & \\
MR imaging ( \pm CT) & $92 \%(189 / 205)$ \\
CT only & $8 \%(16 / 205)$ \\
Conventional vascular imaging & \\
$\quad$ (cervical-cerebral) & \\
CTA only & $18 \%(36 / 205)$ \\
MRA only & $37 \%(75 / 205)$ \\
CTA and MRA & $23 \%(47 / 205)$ \\
CTA and DSA & $5 \%(11 / 205)$ \\
MRA and DSA & $10 \%(20 / 205)$ \\
CTA, MRA, and DSA & $8 \%(16 / 205)$ \\
Cardiac investigations & \\
Electrocardiography & $100 \%(205 / 205)$ \\
Rhythm monitoring $\geq 24$ hours & $29 \%(59 / 205)$ \\
Transthoracic echocardiography & $68 \%(140 / 205)$ \\
Transesophageal echocardiography & $6 \%(30 / 205)$ \\
Laboratory investigations & \\
Serology screen for vasculitis & $25 \%(51 / 205)$ \\
Serology screen for & $34 \%(69 / 205)$ \\
hypercoagulability & \\
CSF analysis & $14 \%(28 / 205)$ \\
Brain biopsy & $2 \%(4 / 205)$ \\
\hline
\end{tabular}

The 2 stroke neurologists then reviewed the VW-MR imaging interpretation for each case they had previously classified, and considering the VW-MR imaging findings in the context of the entire conventional stroke work-up, they each independently either confirmed or reclassified the stroke etiology for each patient.

\section{Statistical Analysis}

Analysis software was SPSS, Version 24 (IBM, Armonk, New York). We calculated an unweighted Cohen $\kappa$ statistic to measure interobserver agreement for categorization of VW-MR imaging findings, classification based on conventional work-up, and classification incorporating $\mathrm{VW}-\mathrm{MR}$ imaging. We calculated the overall proportion of patients with etiologic classifications altered by intracranial VW-MR imaging and then compared the proportion of patients in each etiologic category based on conventional work-up versus conventional work-up supplemented with VWMR imaging and used the McNemar test to identify significant differences. To identify factors from the conventional work-up (age, sex, time from symptom onset to VW-MR imaging, etiologic classification) that were associated with a change in etiologic classification after VW-MR imaging, we performed univariate logistic regression analysis with "change in diagnosis after VWMR imaging" as the dependent variable, followed by an exploratory multivariate logistic regression.

\section{RESULTS}

\section{Patients Characteristics and Conventional Work-Up}

The study included 205 patients: 187 (91\%) with stroke and 18 (9\%) with TIA. Ischemic events were in the anterior circulation in 123 patients $(60 \%)$, the posterior circulation in 64 patients (31\%), and both in 18 patients (9\%). The median age was 56 years (interquartile range $=44-67$ years), and $51 \%$ of patients $(106 / 205)$ were men. Table 2 describes the conventional stroke investigations. The median time from symptom-onset to VW-MR imaging was 14 days (interquartile range $=5-120$ days). VW-MR imaging quality was nondiagnostic in $3 / 205$ (1.5\%) patients. All 205 patients were included in the analysis.

\section{Expert Panel Interobserver Agreement}

Neuroradiologists' categorizations of VW-MR imaging findings had good ${ }^{15}$ interobserver agreement (Cohen $\kappa=0.75$; 95\% CI, 0.59-0.91). Neurologists' classifications of stroke etiology had good interobserver agreement before incorporating VW-MR imaging (Cohen $\kappa=0.76$; 95\% CI, 0.61-0.90) and very good interobserver agreement after incorporating VW-MR imaging (Cohen $\kappa=0.87$; 95\% CI, 0.74-0.99).

\section{Impact of VW-MR Imaging on Etiologic Classificaion}

The etiologic classification was altered by intracranial VWMR imaging in 55\% (112/205) of patients. The On-line Table provides details. The most common etiologic classification based on the conventional work-up was intracranial arteriopathy not otherwise specified, and 92\% (59/64) of patients in this subgroup had an altered etiologic classification after VW-MR imaging.

VW-MR imaging led to a decrease in the proportion of patients classified as having "intracranial arteriopathy not otherwise specified" from $31 \%$ to $4 \%$ (64/205 versus $9 / 205$; $P<.001)$ and an increase in the proportion of patients classified as having "intracranial atherosclerotic disease" from $23 \%$ to $57 \%$ (48/205 versus $116 / 205 ; P<.001)$. VW-MR imaging led to a decrease in the proportion classified as "etiology undetermined due to 2 or more potential causes" from $4 \%$ to $1 \%$ (9/205 versus $2 / 205 ; P=$ $.016)$, and a decrease in the proportion classified as having "small-vessel occlusion" from $3 \%$ to $0 \%$ (7/205 versus $1 / 205 ; P=$ .031). The Figure shows a representative case.

\section{Predictors of Impact}

In the multivariate analysis, the 1 factor that independently predicted a change in etiologic classification after VW-MR imaging was the conventional work-up classification of intracranial arteriopathy not otherwise specified (odds ratio $=8.9$; 95\% CI, 3.027.2). Factors that independently predicted no change were the conventional work-up classification of intracranial atherosclerotic disease $(\mathrm{OR}=0.2 ; 95 \% \mathrm{CI}, 0.1-0.4)$ or cardioembolism $(\mathrm{OR}=$ $0.1 ; 95 \%$ CI , 0-0.6). Table 3 provides details.

\section{DISCUSSION}

The objective of our study was not to measure the diagnostic accuracy of intracranial VW-MR imaging but to apply the current guidelines for interpretation of VW-MR imaging and measure the impact of the technique on the etiologic classification in patients with recent ischemic stroke or TIA. We found that VWMR imaging substantially increased the proportion of strokes attributed to intracranial atherosclerotic disease. VW-MR imaging did not change the overall proportion of strokes attributed to vasculitis, but it altered which particular strokes were attributed to vasculitis. VW-MR imaging was most likely to have a 


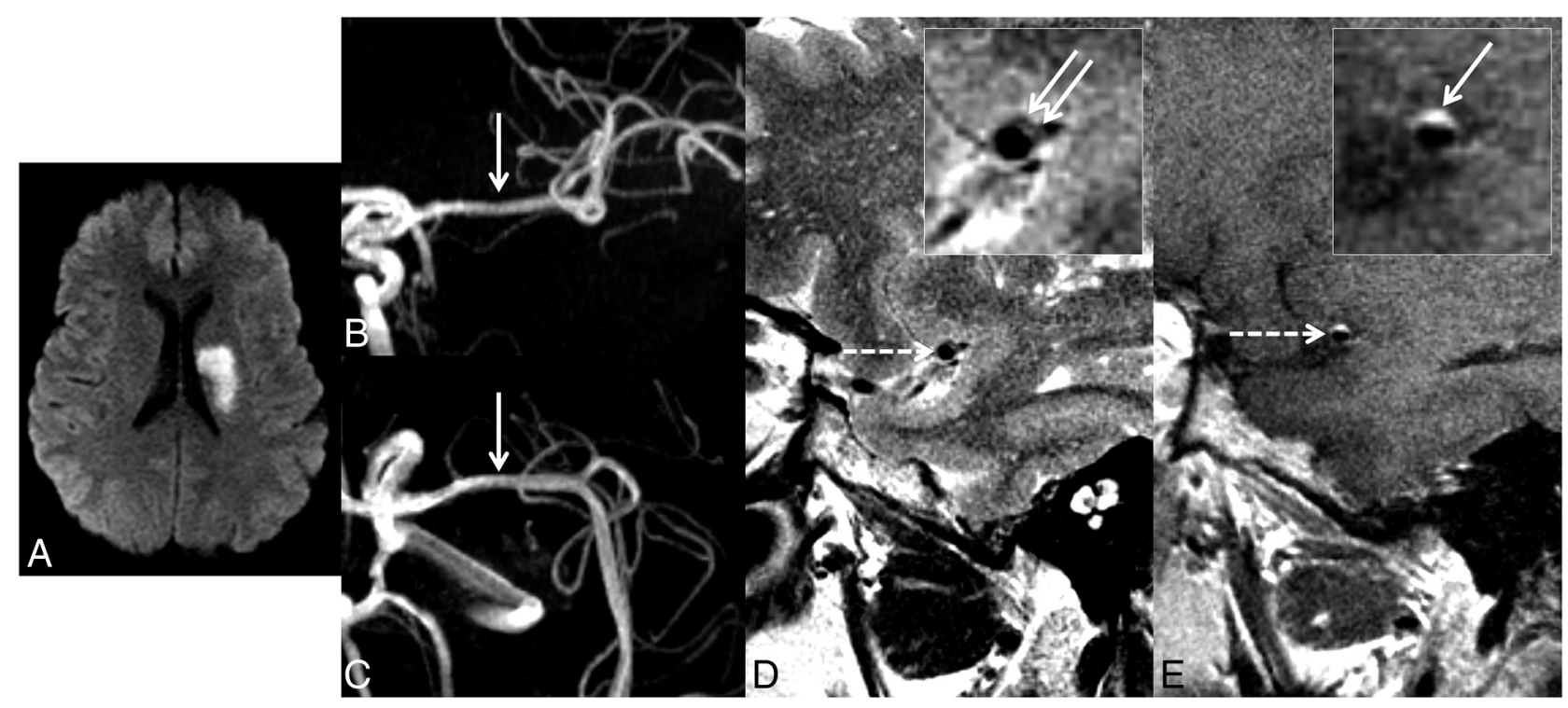

FIGURE. Representative case with conventional stroke work-up with negative findings and altered etiologic classification after VW-MR imaging. Diffusion-weighted MR imaging $(A)$ shows an acute infarct in the left MCA lenticulostriate territory. MRA anterior-posterior $(B)$ and craniocaudal (C) projections show no/minimal narrowing of the left MCA (arrows). Sagittal T2-weighted VW-MR imaging (D) shows a cross-section through the left MCA (dashed arrow), and a magnified view (inset box) shows focal, eccentric, thickening of the superior-posterior wall of the left MCA (solid arrows). Sagittal contrast-enhanced T1-weighted VW-MR imaging (E) shows the same vessel (dashed arrow) with corresponding wall enhancement (solid arrow). The VW-MR imaging appearance is consistent with atherosclerotic plaque, and the enhancement is a finding more common in recently symptomatic plaque. Adapted with permission from Schaafsma et al. ${ }^{23}$

Table 3: Factors associated with revised etiologic classification when conventional stroke work-up is supplemented with VW-MR imaging

\begin{tabular}{|c|c|c|}
\hline & \multicolumn{2}{|c|}{$\begin{array}{l}\text { OR }(95 \% \mathrm{Cl}) \text { for Change in Etiologic } \\
\text { Classification when Conventional Work-Up } \\
\text { is Supplemented with VW-MR Imaging }\end{array}$} \\
\hline & $\begin{array}{l}\text { Univariate Logistic } \\
\text { Regression }\end{array}$ & $\begin{array}{l}\text { Multivariate Logistic } \\
\text { Regression }\end{array}$ \\
\hline Age & $0.97^{\mathrm{a}}(0.95-0.99)$ & $0.99(0.97-1.01)$ \\
\hline Sex & $1.05(0.61-1.82)$ & $1.14(0.55-2.39)$ \\
\hline $\begin{array}{l}\text { Time interval between symptom onset and } \\
\text { VW-MR imaging (days) } \\
\text { Etiologic classification based on conventional } \\
\text { stroke work-up }\end{array}$ & $1.00(1.00-1.00)$ & NA \\
\hline $\begin{array}{l}\text { Intracranial arteriopathy not otherwise } \\
\text { specified }\end{array}$ & $19.59^{\mathrm{a}}(7.39-51.91)$ & $8.94^{\mathrm{a}}(2.95-27.19)$ \\
\hline Intracranial atherosclerotic disease & $0.09^{\mathrm{a}}(0.04-0.20)$ & $0.15^{\mathrm{a}}(0.05-0.41)$ \\
\hline Undetermined etiology & $0.86(0.41-1.82)$ & NA \\
\hline Vasculitis & $1.58(0.56-4.45)$ & $1.39(0.42-4.55)$ \\
\hline Cardioembolism & $0.10^{\mathrm{a}}(0.23-0.46)$ & $0.12^{\mathrm{a}}(0.02-0.57)$ \\
\hline Other determined etiology & $0.48(0.11-2.08)$ & $0.43(0.09-21.83)$ \\
\hline Small-vessel occlusion & $5.21(0.62-44.06)$ & $4.92(0.54-44.73)$ \\
\hline Cervical atherosclerotic disease & $0.55(0.09-3.33)$ & NA \\
\hline Arterial dissection & $1.39 \mathrm{E}+9(0.001$ to $\propto)$ & NA \\
\hline $\begin{array}{l}\text { Reversible cerebral vasoconstriction } \\
\text { syndrome }\end{array}$ & $0.41(0.04-4.95)$ & $0.39(0.03-4.83)$ \\
\hline
\end{tabular}

Note:-NA indicates not applicable as a factor; not included in the multivariate logistic regression.

${ }^{\text {a }}$ Statistically significant.

diagnostic impact for patients who already had categorization as having intracranial arteriopathy based on the conventional workup. The length of time between symptom onset and VW-MR imaging was not a predictor of impact.

We were not surprised to find strokes newly attributed to intracranial atherosclerotic disease after VW-MR imaging. VW-
MR imaging can detect plaque that is occult on conventional vascular imaging, and previous studies have shown plaque on VW-MR imaging in patients with cryptogenic lacunar infarction. ${ }^{3,4}$ This is important because symptomatic intracranial plaque portends a high risk of recurrent stroke and can prompt specific treatment such as dual antiplatelet drug therapy and optimization of blood pressure and lipid profile. ${ }^{16}$ However, the presence of intracranial atherosclerotic plaque does not alone imply that intracranial plaque is the culprit, and we will return to this point later in this discussion.

VW-MR imaging did not alter the proportion of strokes attributed to vasculitis, but it altered which particular strokes were attributed to vasculitis. Among patients categorized as having vasculitis based on conventional work-up, only two-thirds were categorized as having vasculitis after VW-MR imaging. Most of these patients had a vessel wall lesion with typical characteristics of atherosclerotic plaque. Conversely, among patients categorized as having vasculitis after VW-MR imaging, only one-third had been categorized as having vasculitis based on conventional work-up alone. Most of these patients had been 
categorized as having intracranial arteriopathy not otherwise specified rather than a specific condition by conventional workup alone.

The definitive test for central nervous system vasculitis is brain biopsy. A limitation of biopsy is undersampling due to spatially heterogeneous disease or the need to avoid eloquent regions. ${ }^{5}$ Also, vasculitis may predominate in the larger intracranial arteries rather than the smaller vessels that are usually biopsied. ${ }^{17} \mathrm{VW}$-MR imaging is a noninvasive means to assess arterial wall inflammation, and this can help discriminate between vasculitis and mimics such as reversible cerebral vasoconstriction syndrome and atherosclerotic disease. ${ }^{7,8,12,18}$

We had few patients categorized as having etiology undetermined due to $\geq 2$ potential causes, but VW-MR imaging significantly decreased the proportion of patients in this category. VWMR imaging can be useful in this context because it provides information on disease activity.

The factor that independently predicted a change in the etiologic classification after VW-MR imaging was the conventional work-up classification as intracranial arteriopathy not otherwise specified. This was not unexpected, but the high odds ratio $(\mathrm{OR}=$ 8.9; 95\% CI, 3.0-27.2) emphasizes that a pre-existing abnormality on conventional imaging increases the likelihood of impact from VW-MR imaging. We found that the time between symptom onset and VW-MR imaging was not a predictor of impact from VW-MR imaging in our study, which had a median delay of 2 weeks, but it may be best to perform VW-MR imaging within weeks rather than months of symptom onset because findings such as atherosclerotic plaque enhancement may wane after several weeks to months. ${ }^{19,20}$

There is no single, ideal reference standard for determining stroke etiology. Therefore, we modelled our study design on clinical practice and used an expert panel to categorize stroke etiology. The stroke neurologists integrated multiple clinical, laboratory, and imaging factors to decide on the categorization. For example, 1 patient had both a patent foramen ovale and an intracranial atherosclerotic plaque, so the neurologists had to decide which one was the likely etiology. For this case, the plaque was designated as the likely etiology because contrast echocardiography had characterized the patent foramen ovale as "low-risk," intracranial plaque was only evident in the artery supplying the territory of the stroke, and the plaque was intensely enhancing (a feature that the VW-MR imaging framework views as a feature of recently symptomatic plaque). ${ }^{19,20}$ We believe this use of an expert panel, with independent assessments and measurement of in-terobserver agreement, is a re-asonable approach to measure the diagnostic impact of VW-MR imaging. However, it is important to recognize the potential for confirmation bias: interpreting the additional information provided by VW-MR imaging as overly definitive.

This study used T1-, T2-, and contrast-enhanced T1-weighted VW-MR imaging sequences, and the interpreting neuroradiologists used the multiple tissue-weighting and multiple imaging planes to confirm the vessel wall findings as recommended. ${ }^{9}$ Some centers use only T1-weighted VW-MR imaging sequences. Our local preference has been to routinely include a T2-weighted sequence as well because it can help confirm that a finding is within the vessel wall rather than within the lumen and can contribute to the characterization of vessel wall lesions (eg, identification of hyperintensity within the fibrous cap of atherosclerotic plaque).

We evaluated patients who were referred for VW-MR imaging to clarify the cause of a stroke or TIA. We believe this is a clinically relevant patient group because it likely approximates the types of patients who will have intracranial VW-MR imaging at other centers. However, relying on the referral patterns of multiple stroke neurologists who have different thresholds for requesting intracranial VW-MR imaging does introduce a selection bias, and the proportion of patients with altered etiologic classifications after VW-MR imaging may differ at other institutions.

We used a modified TOAST ${ }^{14}$ classification system because this system is well-known and reflects the kind of etiologic classification often used in clinical practice, but there are other systems we could have used, ${ }^{21,22}$ and these may have led to different proportions of patients with a revised etiologic classification. Also, we did not attempt to stratify the level of diagnostic confidence within each particular etiologic category, but changes in the level of confidence within categories may affect therapeutic decisionmaking, too.

All patients had electrocardiography and many (74\%) had echocardiography, but fewer (29\%) had Holter rhythm monitoring for $>24$ hours. This scenario likely reflects the high proportion of patients who had intracranial arteriopathy not otherwise specified or a working diagnosis based on conventional work-up rather than completely cryptogenic stroke.

\section{CONCLUSIONS}

We found that when VW-MR imaging is performed to clarify the etiology of a stroke or TIA, it can have a substantial impact on etiologic classification. Because this impact is substantial, intracranial VW-MR imaging has the potential to improve therapeutic decision-making for many patients. The contrary is also true: Improper application of the interpretive framework or limitations of the framework itself have the potential to misinform therapeutic decision-making for many patients. Physicians who are performing and interpreting VW-MR imaging should familiarize themselves with technical recommendations and interpretive pitfalls for VW-MR imaging and recognize that there remain gaps in knowledge and that research is ongoing.

\section{ACKNOWLEDGMENTS}

We appreciate the dedication and skill of the Toronto Western Hospital MR imaging technologists involved in this work, especially Nicole Bennett, Holly Chun, Colleen Grace, Mary Hatchard, Olga Ioffina, Nisha James, Mahnaz Miraftabi, Asma Naheed, Jessica Pinto, Keith Ta, and Danfeng Zhao.

Disclosures: Daniel M. Mandell—RELATED: Grant: Ontario Academic Health Science Centers Innovation Fund.* *Money paid to the Institution.

\section{REFERENCES}

1. Saver JL. Cryptogenic stroke. N Engl J Med 2016;374:2065-74 CrossRef Medline

AJNR Am J Neuroradiol 40:1701-06 Oct 2019 www.ajnr.org 
2. Chung JW, Kim BJ, Sohn CH, et al. Branch atheromatous plaque: a major cause of lacunar infarction (high-resolution MRI study). Cerebrovasc Dis Extra 2012;2:36-44 CrossRef Medline

3. Yoon Y, Lee DH, Kang DW, et al. Single subcortical infarction and atherosclerotic plaques in the middle cerebral artery: high-resolution magnetic resonance imaging findings. Stroke 2013;44:2462-67 CrossRef Medline

4. Klein IF, Lavallée PC, Mazighi M, et al. Basilar artery atherosclerotic plaques in paramedian and lacunar pontine infarctions: a high-resolution MRI study. Stroke 2010;41:1405-09 CrossRef Medline

5. Powers WJ. Primary angiitis of the central nervous system: diagnostic criteria. Neurol Clin 2015;33:515-26 CrossRef Medline

6. Swartz RH, Bhuta SS, Farb RI, et al. Intracranial arterial wall imaging using high-resolution 3-Tesla contrast-enhanced MRI. Neurology 2009;72:627-34 CrossRef Medline

7. Mandell DM, Matouk CC, Farb RI, et al. Vessel wall MRI to differentiate between reversible cerebral vasoconstriction syndrome and central nervous system vasculitis: preliminary results. Stroke 2012;43:860-62 CrossRef Medline

8. Mossa-Basha M, Hwang WD, De Havenon A, et al. Multicontrast high-resolution vessel wall magnetic resonance imaging and its value in differentiating intracranial vasculopathic processes. Stroke 2015;46:1567-73 CrossRef Medline

9. Mandell DM, Mossa-Basha M, Qiao Y, et al. Intracranial vessel wall MRI: principles and expert consensus recommendations of the American Society of Neuroradiology. AJNR Am J Neuroradiol 2017;38:218-29 CrossRef Medline

10. Lindenholz A, van der Kolk AG, Zwanenburg JJM, et al. The use and pitfalls of intracranial vessel wall imaging: how we do it. Radiology 2018;286:12-28 CrossRef Medline

11. Mossa-Basha M, de Havenon A, Becker KJ, et al. Added value of vessel wall magnetic resonance imaging in the differentiation of Moyamoya vasculopathies in a non-Asian cohort. Stroke 2016; 47:1782-88 CrossRef Medline

12. Mossa-Basha M, Shibata DK, Hallam DK, et al. Added value of vessel wall magnetic resonance imaging for differentiation of nonocclusive intracranial vasculopathies. Stroke 2017;48:3026-33 CrossRef Medline
13. Dieleman $N$, van der Kolk AG, Zwanenburg JJ, et al. Imaging intracranial vessel wall pathology with magnetic resonance imaging: current prospects and future directions. Circulation 2014;130:192201 CrossRef Medline

14. Adams HP Jr, Bendixen BH, Kappelle LJ, et al. Classification of subtype of acute ischemic stroke: definitions for use in a multicenter clinical trial-TOAST. Trial of Org 10172 in Acute Stroke Treatment. Stroke 1993;24:35-41 CrossRef Medline

15. Brennan P, Silman A. Statistical methods for assessing observer variability in clinical measures. BMJ 1992;304:1491-94 CrossRef Medline

16. Qureshi AI, Caplan LR. Intracranial atherosclerosis. Lancet 2014; 383:984-98 CrossRef Medline

17. Rossi CM, Di Comite G. The clinical spectrum of the neurological involvement in vasculitides. J Neurol Sci 2009;285:13-21 CrossRef Medline

18. Singhal AB, Topcuoglu MA, Fok JW, et al. Reversible cerebral vasoconstriction syndromes and primary angiitis of the central nervous system: clinical, imaging, and angiographic comparison. Ann Neurol 2016;79:882-94 CrossRef Medline

19. Gupta A, Baradaran H, Al-Dasuqi K, et al. Gadolinium enhancement in intracranial atherosclerotic plaque and ischemic stroke: a systematic review and meta-analysis. J Am Heart Assoc 2016;5 CrossRef Medline

20. Skarpathiotakis M, Mandell DM, Swartz RH, et al. Intracranial atherosclerotic plaque enhancement in patients with ischemic stroke. AJNR Am J Neuroradiol 2013;34:299-304 CrossRef Medline

21. Amarenco P, Bogousslavsky J, Caplan LR, et al. The ASCOD phenotyping of ischemic stroke (updated ASCO phenotyping). Cerebrovasc Dis 2013;36:1-5 CrossRef

22. Ay H, Benner T, Arsava EM, et al. A computerized algorithm for etiologic classification of ischemic stroke: the Causative Classification of Stroke System. Stroke 2007;38:2979-84 CrossRef Medline

23. Schaafsma JD, Mikulis DJ, Mandell DM. Intracranial vessel wall MR imaging: an emerging technique with a multitude of uses. Top Magn Reson Imaging 2016;25:41-47 CrossRef Medline 
$\mathrm{n}$ the article commentary "Impact of Vessel Wall MR Imaging in the Work-Up for Ischemic Stroke" (Song JW. AJNR Am J Neuroradiol 2019;40:1707-08. 10.3174/ajnr.A6241), the author omitted a citation for the article discussed. The referenced article is "Diagnostic Impact of Intracranial Vessel Wall MRI in 205 Patients with Ischemic Stroke or TIA" (Schaafsma JD, Rawal S, Coutinho JM, et al. AJNR Am J Neuroradiol 2019;40:1701-06. 10.3174/ajnr.A6202).

http://dx.doi.org/10.3174/ajnr.A6353 